\title{
Developing Markerless Augmented Reality for Furniture Mobile Application
}

\author{
Raj Raval $^{\text {a }}$, Tarun Sankhla ${ }^{\mathrm{a}}$, Rushabh Shah ${ }^{\mathrm{a}}$, Swati Nadkarni ${ }^{\mathrm{a}}$ \\ ${ }^{a}$ Shah \& Anchor Kutchhi Engineering College, Mumbai-400088, India \\ Email: raj.raval@sakec.ac.in
}

Received: 10 July 2020; Accepted: 13 November 2020; Published: 08 February 2021

\begin{abstract}
In India, purchasing furniture online consistently faces a quandary regarding how one can comprehend whether the furniture is a solid match to purchase, regardless of whether it will fit in space without really contacting and looking at the furniture in detail. With such a dilemma there are chances for online stores to decline in their business. There is always a good certainty that technology can help to bridge the barrier and help one to overcome the dilemma. This study creates a unique light on how Augmented Reality can help to create and simulate buying furniture an online experience and enhancing customer experience provided uniqueness and mobility with the help of a mobile application. Our application allows users to simulate and experience furniture in 3D simulation using markerless augmented reality. Users can try a piece of furniture in their space supported by gestures and color furniture to try out diverse tints of the same. This research advances a new way of implementing markerless augmented reality for buying furniture products online provided by mobile applications and communicating with virtual objects in a real environment with an easy-touse user interface.
\end{abstract}

Index Terms: Augmented Reality, User Experience, Online Shopping, Mobile Application, E-Commerce

\section{Introduction}

In this paper, the major research objective is to show how through augmented reality we aim at simplifying the user's experience and also enhancing the user's perception and interaction with virtual(3D) objects within the real world, in real-time. So the purpose of this research is that purchasing product such as furniture for interior design is a complex and hectic process and not satisfying to the consumer, and one of the major reason for this is the consumer cannot place or fit the furniture according to the space requirement of their place before buying, and the previous augmented realitybased research systems do not allow users to view virtual furniture with precise relative 3D models, motion tracking, and environmental understanding, in the context of the real-time environment and further, they do not allow the user to alter the dimensions and they had marker-based augmented reality approach. So we proposed an alternative solution ie: a mobile application that can be used for interior designing of home furniture through markerless augmented reality; wherein a customer can form a 3D object model of the furniture through which they can easily scale multiple 3D models of furniture object and place them in their space and can alter the color or dimensions of furniture according to their preferences in real-time and then can go on and buy the furniture with the details provided about the furniture in the android application, so the main aim for research in augmented reality is to enhance customer experience and making the 3D model object rendering more realistic and convenient as possible so the end customer is satisfied; which in turn can enhance and boost e-commerce and online shopping of furniture.

So for enhancing the user interaction and resolving limitations; we enforced motion tracking that is used to understand the motion of objects and track their movements and accordingly to that the 3D model will simulate to give a better perspective and also environmental understanding that allows the mobile application to detect the surface and according to its size and inclination such as horizontal, vertical or angled surface the projection of the 3D model will alter, and also estimation of light in the environment such as current light intensity and lighting condition so according to that the 3D model will render its projection and be more realistic and dynamic to the real object in the surrounding environment and make customer interaction more efficient. So we hope to achieve simulation of the furniture object and augment in the real-world environment.

\section{Requirements and Feasibility Analysis}

The application is more optimistic in results and higher mobility which leads to less requirement. Since the application is developed on mobile. It only requires the mobile itself. Alongside the IDEs, it is created on i.e: Android 
Studio which is supported by macOS, Windows, and Linux. It is powered by ARCore Framework provided by Google and it is capable of running on Android OS and iOS since it is a multi-platform framework.

\section{Working and Concept}

The application has a simple interface that helps in providing clarity and interactivity to the user. It uses the camera of the mobile device which plays a vital role by communicating with the application and the framework that provides augmented reality features. A demo application is created that showcases the demonstration and the capabilities that augmented reality can show. We choose the ARCore SDK provided by Google because it is capable of providing Augmented Reality Experience on cross-platform devices.

The application working is divided into 3 parts:

- Working of the ARCore SDK

- Technical Working of the Application

- Working of the Application from the end-user perspective

ARCore SDK provides three key features that help developers to create a great user-experience in augmented reality. They are as follows:

- Motion Tracking

- Environmental Understanding

- Light Estimation

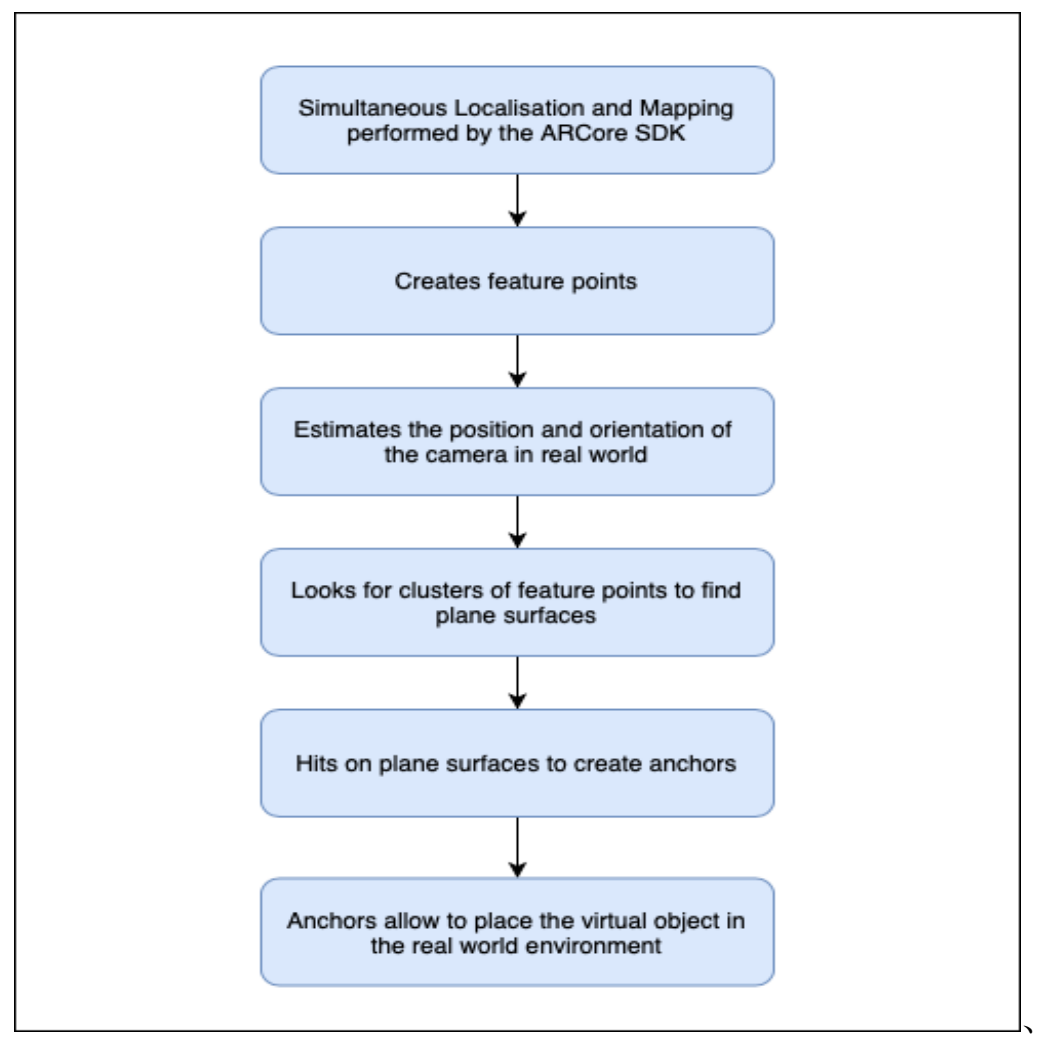

Fig.1. ARCore SDK Core Functioning to help the app provide the AR Experience

Motion Tracking: The framework uses a technology called simultaneous localization and mapping (SLAM) for understanding the devices' relative positioning in the world. It then creates points from the captured image by the camera known as the feature points which then help to compute the changes while moving the device around the world. With the help of the feature points, it provides to creates hits on the feature points to place the $3 \mathrm{D}$ objects in the form of anchors

Environmental Understanding: It continuously learns the environment and finds feature points and planes (horizontal/vertical/angular) ie: walls, tables, and angular slopes. By creating the features points with the help of motion tracking, it allows placing objects on the recognized planes. 
Light Estimation: The framework provides the ability to provide lighting to the 3D Objects with intensity and color correction with the help of the given camera captured image. Even in a night/dark environment, it provides the ability to provide lighting and intensity to the virtual object to make it feel in a realistic environment.

The above figure showcases how the ARCore Sceneform SDK works in the application that helps to provide an interactive augmented reality experience with the virtual objects. Firstly it begins with motion tracking with the help of the camera image, it performs SLAM (Simultaneous Localisation and Mapping) process which helps to understand the environment and creates feature points on the surface it can detect properly. It calculates and estimates the position and orientation of the camera in the real-world and looks for a cluster of features points to perform hit tests to create anchors that will place virtual objects in the real-world environment according to the position and orientation estimated by the camera. This is how the ARCore SDK core functioning works and helps the application to provide such an experience.

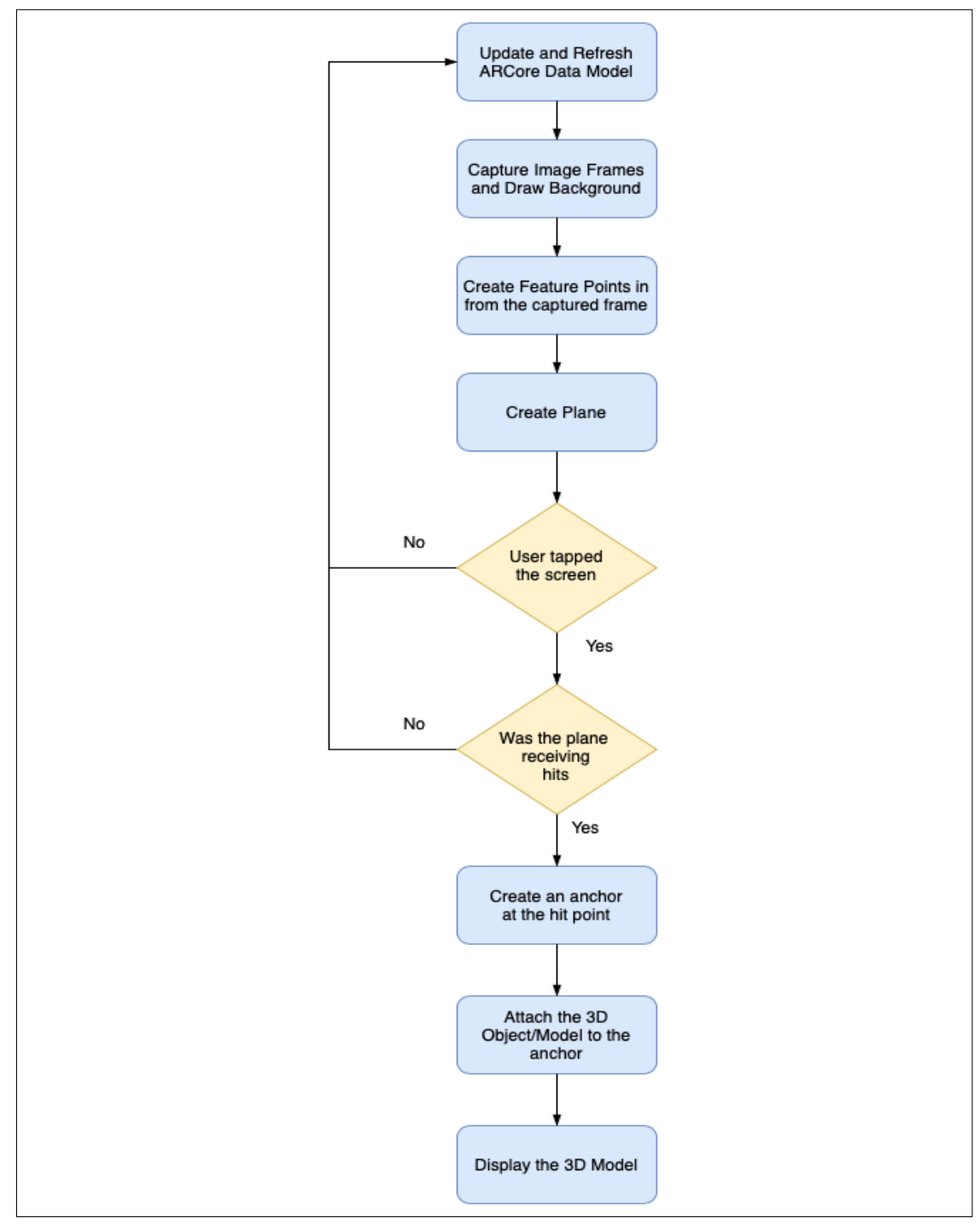

Fig.2. Technical working of the application

The above figure showcases the technical working of the application on how it works with the help of the ARCore Sceneform SDK. The mobile camera is considered to be an essential part of working in providing interaction with virtual objects in the real world. The application provides an AR View allowing the virtual objects to place in the environment. With the help of the camera, the data model provided by ARCore refreshes to capture image frames and detect to create feature points which allow to find the plane and allow the user to tap to perform a hit and the hit result is created by understanding the orientation and position of the hit created by tapping on the image frame captured by the camera, it then creates the anchor from the hit result and position provided and it allows to place the 3D Furniture Model to place it at the given anchor. The app also allows users to change the color of the 3D Object and the app does this by changing the material of the 3D Object. 


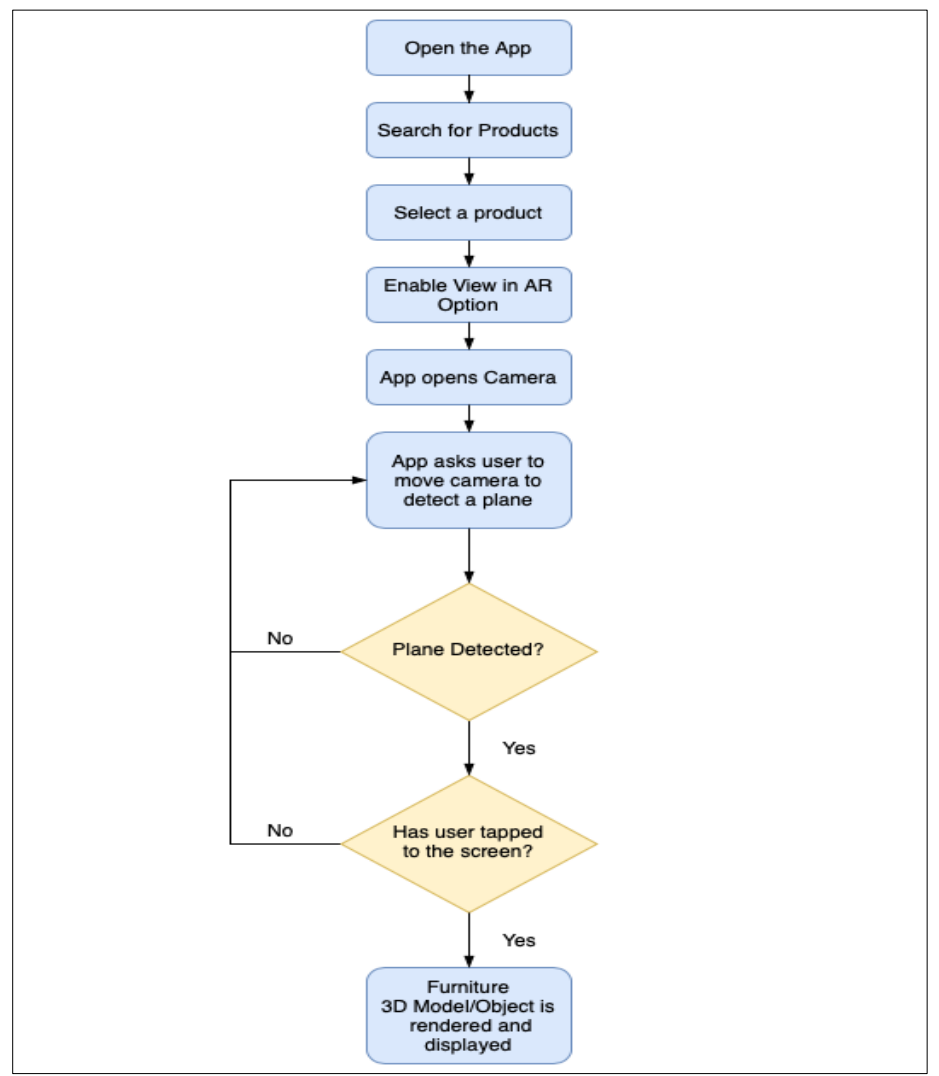

Fig.3. Working of the application from the end-user perspective

From an end-user perspective, the app appears to be an e-commerce product and the user searches and selects a product to view the information, the app provides a View-In-AR call to action where the app opens up an AR View and allows user to find a plane and tap on the screen to perform a hit test to place the anchor and interact with the virtual object in the real-world environment, the user can also be able to change the color of the furniture as per convenience and also add multiple models for a better experience.

\section{Application Screenshots}

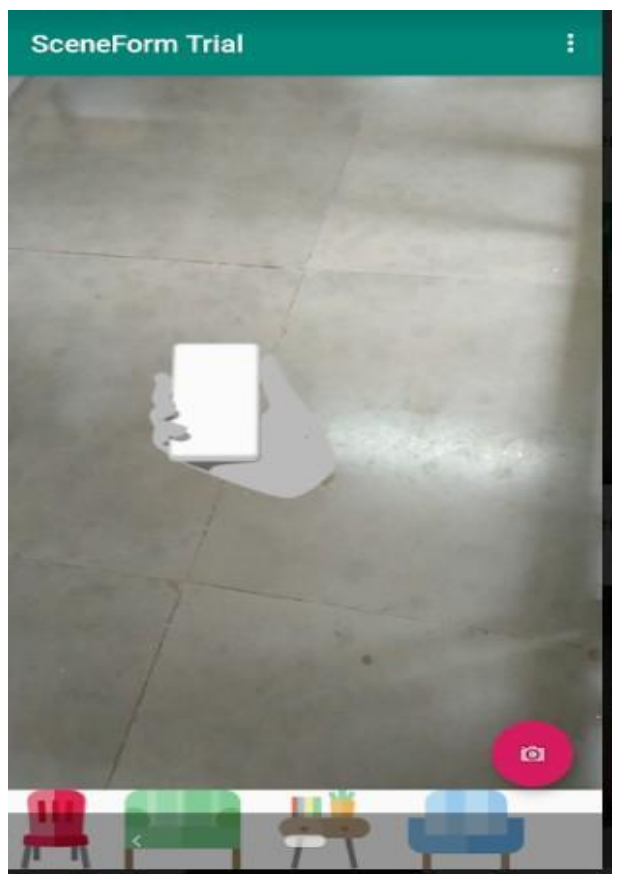

Fig.4. Application detecting a plane through understanding the environment - Playground View 


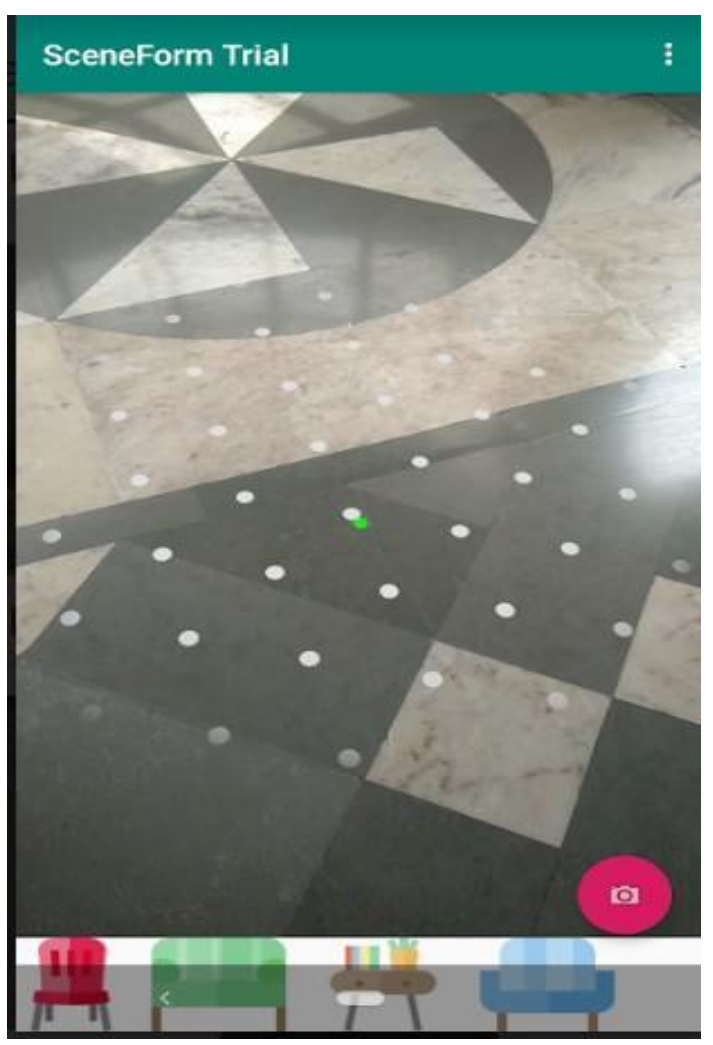

Fig.5. Application has detected plane through understanding the environment and shows users - Playground View

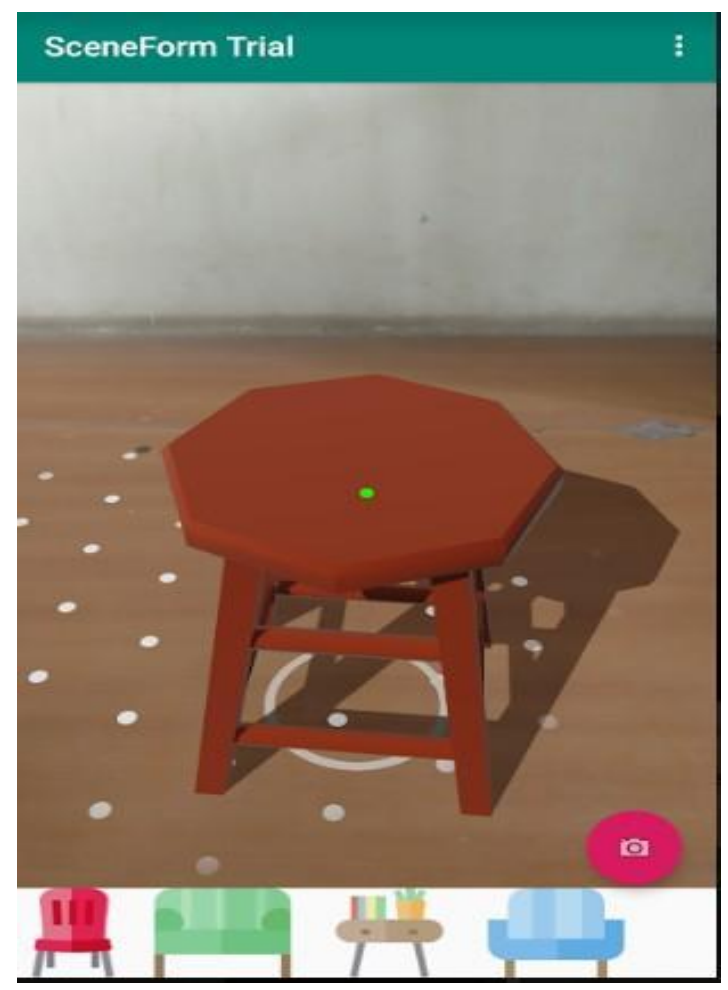

Fig.6. Application rendered the 3D model - Playground View 


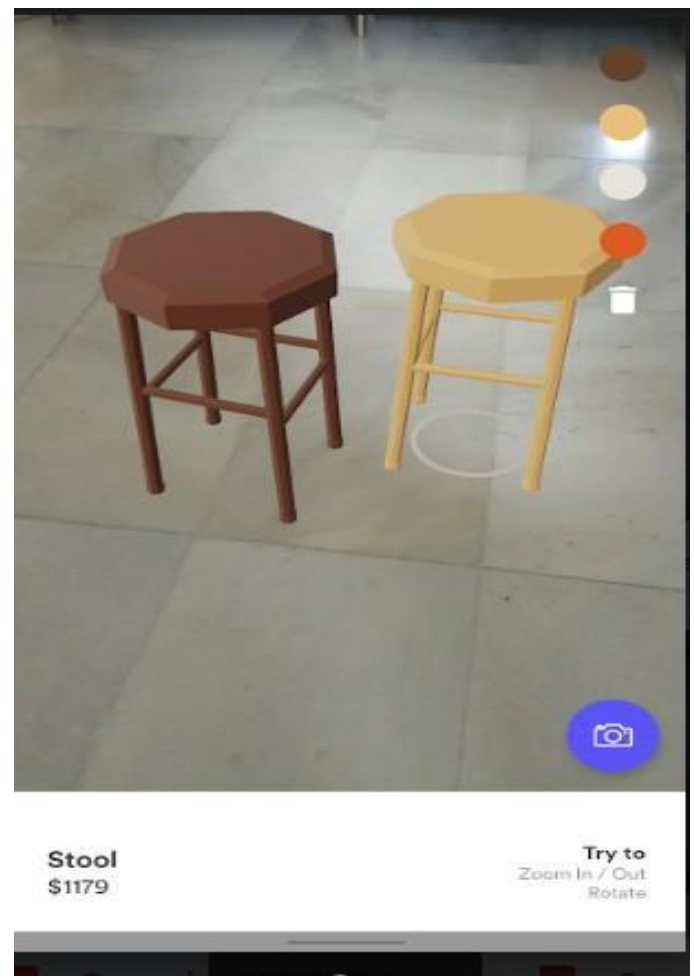

Fig.7. Application has the ability to change color of the furniture product for concise furniture buying atmosphere

\section{Conclusion}

The research concludes and states how Augmented Reality can not only leverage online shopping ecosystems but also boost user interaction as it provides interaction with products in the real world environment that leads to catering products with better user experience and mobility. Due to an increase in the adaptation of online shopping in recent years. The application can play a vital role and provide a unique selling point for businesses to grow in their vertical. Many businesses have started to adapt this to their work-related purposes such as interior decoration and design, decoration, and home-planning. As mobile phones are coming equipped with better camera features like depth interaction, augmented reality drawing support and LiDAR scanners, there will be huge growth in the adaptation of markerless augmented reality mobile applications and the application is a starting point in product innovation for online e-commerce businesses and providing a noteworthy edge of advancement in this domain of technology.

\section{Acknowledgements}

We would like to express our special thanks of gratitude to our Principal Dr. Bhavesh Patel for his constant encouragement and gave us the golden opportunity to do this wonderful project based on Augmented Reality, which also helped us in doing a lot of Research, and we came to know about so many new things we are really thankful to them. We would also like to thank the Review Committee for their invaluable suggestions and feedback without whom our work would have been very difficult.. The blessing, help and guidance given by his time to time shall carry us a long way in the journey of life on which we are about to embark.

\section{References}

[1] Springer - An Interactive Augmented Reality Furniture Customisation System - Tzu-Chien Young,Shana Smith.

[2] 2016 11th International Conference on Computer Science \& Education (ICCSE) - Research on the augmented reality system without identification markers for home exhibition - Liyan Chen, Xiaoyuan PengJunfeng Yao, Hong Qiguan, Chen Chen, Yihan Ma.

[3] 2018 International Conference on Smart City and Emerging Technology (ICSCET)- IEEE Xplore - Markerless Augmented Reality based Interior Designing System - Santosh Sharma, Yash Kaikini, Parth Bhodia, Sonali Vaidya.

[4] Journal of Business Research Volume 116, August 2020 -ScienceDirect - The playground effect: How augmented reality drives creative customer engagement - Alexander Jessen,Tim Hilken, Mathew Chylinski,Dominik Mahr Jonas Heller, Debbie Isobel Keeling, Kode Ruyter.

[5] Department of Industrial Design, National United University, Springer - Gesture-Based Human-Machine Interface: A Case Study Comparing the Realism of Furniture Products in E-Commerce -Kuen-Meau Chen. 
[6] 2017 2nd IEEE International Conference on Recent Trends in Electronics, Information \& Communication Technology (RTEICT) - IEEE Xplore - A study of the factors impacting the adoption of augmented reality in online purchases in India Saikat Chakraborty, Deepak Gupta.

[7] 2018 IEEE International Conference on Teaching, Assessment, and Learning for Engineering (TALE)- IEEE Xplore - Virtual Lab Using Markerless Augmented Reality - Uses ARCore SDK -M.T. Abhishek, P.S. Aswin, Nihal C. Akhil, Ali Souban, Shafeeque K. Muhammedali, Alanna Vial.

[8] Procedia Computer Science, Volume 172, 2020 - ScienceDirect -Prospects of Augmented Reality in Physical Stores using Shopping Assistance App - Ashok kumar.P, Murugavel.R.

[9] Computers in Human Behavior, Volume 101, December 2019 - ScienceDirect - Shopping in the digital world: Examining customer engagement through augmented reality mobile applications - Graeme McLean, Alan Wilson.

[10] 2014 IEEE International Symposium on Mixed and Augmented Reality (ISMAR) - IEEE Xplore - A complete interior design solution with diminished reality - Sanni Siltanen, Henrikki Saraspää, Jari Karvonen.

[11] 2018 IEEE 5th International Conference on Smart Instrumentation, Measurement and Application (ICSIMA)- IEEE Xplore Augmented Reality Application for Architects and interior designers: Interno A cost effective solution - Sidra Nasir , Mohammad Noman Zahid, Talha Ahmed Khan. Kushsairy Kadir, Sheroz Khan.

[12] 2015 Sixth International Conference on Intelligent Systems Design and Engineering Applications (ISDEA)- IEEE Xplore Approach to the Interior Design Using Augmented Reality Technology --Jiang Hui.

[13] International Conference on Virtual and Mixed Reality - Springer - Advanced Interaction Techniques for Augmented Reality Application - Mark Billinghurst, Hirokazu Kato, Seiko Myojin.

[14] International Conference on Virtual, Augmented and Mixed Reality -VAMR 2014: Springer - 3D Design for Augmented Reality - Ivar Kjellmo.

\section{Authors' Profiles}

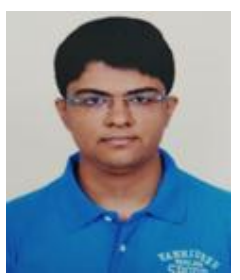

Mr.Raj Raval is pursuing his Bachelor Degree of Engineering in the field of Information Technology Shah and Anchor Kutchhi Engineering. His research interests include Software Engineering, Programming Languages and Application Development, Product Design and Augmented Reality.He also reached the final level of Smart India Hackathon for 2018 and 2019 (Software Edition) and International Blockchain Hackathon by IET. He also has work experience in the corporate sector. He has also given talks at Google Developer Group on Augmented Reality and Figma Community for Design Sprints.

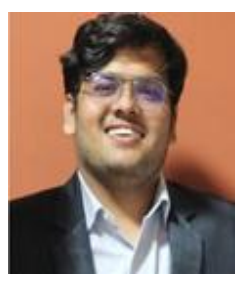

Mr.Tarun Sankhla is pursuing his Bachelor Degree in Engineering in the field of Information Technology Shah and Anchor Kutchhi Engineering. His research interest includesSoftware Engineering and Programming, Augmented Reality,Big Data Integration, Modeling and Management Systems with certification from UC San Diego. He also reached the final level of the Smart India Hackathon 2019 (Software Edition) and International Blockchain Hackathon by IET. He also has work experience in the corporate sector under Mobile Application Development and Web Development.

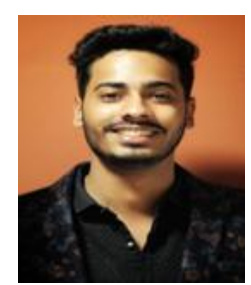

Mr.Rushabh Shah is pursuing his Bachelor Degree of Engineering in the field of Information Technology Shah and Anchor Kutchhi Engineering (SAKEC). His research interest includes Software Engineering and Programming, Database Management. He also reached the final level of Smart India Hackathon (Software Edition) and International Blockchain Hackathon by IET. He also has work experience in the corporate sector .

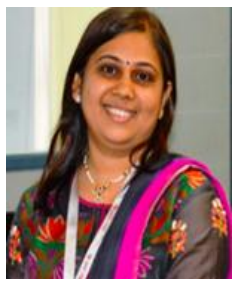

Ms.Swati Nadkarni is an Associate Professor and Head of Information Technology Department in Shah \& Anchor Kutchhi Engineering College (SAKEC). She has 22 years of experience. Her area of specialization includes Machine Learning, Digital Signal and Image Processing, Analog and Digital Circuits. She is the faculty sponsor of SAKEC ACM. She is currently the President of IIC 2.0 in SAKEC. 
How to cite this paper: Raj Raval, Tarun Sankhla, Rushabh Shah, Swati Nadkarni, "Developing Markerless Augmented Reality for Furniture Mobile Application", International Journal of Education and Management Engineering (IJEME), Vol.11, No.1, pp. 11-18, 2021. DOI: $10.5815 / \mathrm{ijeme} .2021 .01 .02$ 